\title{
A new hierarchy for automaton semigroups
}

\author{
Laurent Bartholdi ${ }^{1}$, Thibault Godin ${ }^{2}$, Ines Klimann ${ }^{3}$, and Matthieu Picantin ${ }^{3}$ \\ 1 École Normale Supérieure, Paris, and Georg-August-Universität zu Göttingen, \\ laurent.bartholdi@ens.fr, \\ ${ }^{2}$ University of Turku, Turku, \\ thibault.godin@utu.fi, supported by the Academy of Finland grant 296018 \\ 3 IRIF, UMR 8243 CNRS \& Université Paris Diderot, \\ klimann@irif.fr, picantin@irif.fr
}

\begin{abstract}
We define a new strict and computable hierarchy for the family of automaton semigroups, which reflects the various asymptotic behaviors of the state-activity growth. This hierarchy extends that given by Sidki for automaton groups, and also gives new insights into the latter. Its exponential part coincides with a notion of entropy for some associated automata.

We prove that the ORDER PROBLEM is decidable when the state-activity is bounded. The ORDER Problem remains open for the next level of this hierarchy, that is, when the state-activity is linear. Gillibert showed that it is undecidable in the whole family.

The former results are implemented and will be available in the GAP package FR developed by the first author.
\end{abstract}

Keywords: automaton $\cdot$ semigroup $\cdot$ entropy $\cdot$ hierarchy $\cdot$ decision problem

\section{Introduction}

The family of automaton groups and semigroups has provided a wide playground to various algorithmic problems in computational (semi)group theory [1,2,3,4,5,6,8,9,10,11]. While many undecidable questions in the world of (semi)groups remain undecidable for this family, the underlying Mealy automata provide a combinatorial leverage to solve the WORD Problem for this family, and various other problems in some important subfamilies. Recall that a Mealy automaton is a letter-toletter, complete, deterministic transducer with same input and output alphabet, so each of its states induces a transformation from the set of words over its alphabet into itself. Composing these Mealy transformations leads to so-called automaton (semi)groups, and the Word Problem can be solved using a classical technique of minimization.

The ORDer Problem is one of the current challenging problems in computational (semi)group theory. On the one hand, it was proven to be undecidable for automaton semigroups by Gillibert [8]. On the other hand, Sidki introduced 
a polynomial hierarchy for invertible Mealy transformations in [19] and, with Bondarenko, Bondarenko, and Zapata in [6], solved the Order Problem for its lowest level (bounded invertible automata).

Our main contributions in this paper are the following: an activity-based hierarchy for possibly non-invertible Mealy transformations (Section 3), extending Sidki's construction [19] to non-necessarily-invertible transformations; and a study of the algorithmic properties in the lowest level of the hierarchy, namely transducers with bounded activity. We prove:

Theorem (see Section 5). The Order Problem is decidable for bounded Mealy transformations; namely, there is an algorithm that, given a bounded initial Mealy automaton, decides whether the transformation $\tau$ that it defines has infinite order, and if not finds the minimal $r>s$ such that $\tau^{r}=\tau^{s}$.

Our strategy of proof follows closely that of Sidki's [19] and Bondarenko, Bondarenko, Sidki and Zapata [6], with some crucial differences. On the one hand, a naive count of the number of non-trivial states of a transformation does not yield a useful invariant, nor a hierarchy stable under multiplication; on the other hand, the structure of cyclic semigroups $\left(\left\langle a \mid a^{m}=a^{m+n}\right\rangle\right.$ has index $m$ and period $\left.n\right)$ is more complex than that of cyclic groups $\left(\left\langle a \mid a^{m}\right\rangle\right.$ has order $\left.m\right)$.

\section{Notions from automata and graph theory}

This section gathers some basics about automata, especially some links between automata, Mealy automata, automaton semigroups, and finite-state transformations. We refer the reader to handbooks for graph theory [15], automata theory [16], and automaton (semi)groups [5].

A non-deterministic finite-state automaton (NFA for short) is given by a directed graph with finite vertex set $Q$, a set of edges $\Delta$ labeled by an alphabet $\Sigma$, and two distinguished subsets of vertices $I \subseteq Q$ and $F \subseteq Q$. The vertices of the graph are called states of the automaton and its edges are called transitions. The elements of $I$ and $F$ are called respectively initial and final states. A transition from the state $p$ to the state $q$ with label $x$ is denoted by $p \stackrel{x}{\longrightarrow} q$.

A NFA is deterministic - DFA for short-(resp. complete) if for each state $q$ and each letter $x$, there exists at most (resp. at least) one transition from $q$ with label $x$. Given a word $\mathbf{w}=w_{1} w_{2} \cdots w_{n} \in \Sigma^{*}$ (where the $w_{i}$ are letters), a run with label $\mathbf{w}$ in an automaton (NFA or DFA) is a sequence of consecutive transitions

$$
q_{1} \stackrel{w_{1}}{\longrightarrow} q_{2} \stackrel{w_{2}}{\longrightarrow} q_{3} \rightarrow \cdots \rightarrow q_{n} \stackrel{w_{n}}{\longrightarrow} q_{n+1} .
$$

Such a run is successful whenever $q_{1}$ is an initial state and $q_{n+1}$ a final state. A word in $\Sigma^{*}$ is recognized by an automaton if it is the label of at least one successful run. The language recognized by an automaton is the set of words it recognizes. A DFA is coaccessible if each state belongs to some run ending at a final state. 
Let $\mathcal{A}$ be a NFA with stateset $Q$. The Rabin-Scott powerset construction [14] returns, in a nutshell, the (co)accessible DFA — denoted by $\operatorname{det}(\mathcal{A})$ - with states corresponding to subsets of $Q$, whose initial state is the subset of all initial states of $\mathcal{A}$ and whose final states are the subsets containing at least on final state of $\mathcal{A}$; its transition labeled by $x$ from a state $S \subseteq 2^{Q}$ leads to the state $\{q \mid \exists p \in S, p \stackrel{x}{\rightarrow}$ $q$ in $\mathcal{A}$ \}. Notice that the size of the resulting DFA might therefore be exponential in the size of the original NFA.

The language of a NFA is the subset of $\Sigma^{*}$ consisting in the words recognized by it. Given a language $L \subseteq \Sigma^{*}$, its entropy is

$$
h(L)=\lim _{\ell \rightarrow \infty} \frac{1}{\ell} \log \#\left(L \cap \Sigma^{\ell}\right) .
$$

This quantity appears in various situations, in particular for subshifts [13] and finite-state automata [7]. We shall see how to compute it with matrices.

To a NFA $\mathcal{A}$, associate its transition matrix $A=\left\{A_{i, j}\right\}_{i, j} \in \mathbb{N}^{n \times n}$ where $A_{i, j}$ is the number of transitions from $i$ to $j$. Let furthermore $v \in \mathbb{N}^{n}$ be the row vector with ' 1 ' at all positions in $I$ and $w \in \mathbb{N}^{n}$ be the column vector with ' 1 ' at all positions in $F$. Then $v A^{\ell} w$ is the number of successful runs in $\mathcal{A}$ of length $\ell$. Assuming furthermore that $\mathcal{A}$ is deterministic, $v A^{\ell} w$ is the cardinality of $L \cap \Sigma^{\ell}$. Since the transition matrix of an automaton $\mathcal{A}$ is non-negative, it admits a positive real eigenvalue of maximal absolute value, which is called its PerronFrobenius eigenvalue and is written $\lambda(\mathcal{A})$. Assuming therefore that $\mathcal{A}$ is coaccessible, we get

Proposition 2.1. [18, Theorem 1.2] Let $\mathcal{A}$ be a coaccessible DFA recognizing the language $L$. Then we have $h(L)=\log \lambda(\mathcal{A})$.

\subsection{Mealy transducers}

A Mealy automaton is a DFA over an alphabet of the form $\Sigma \times \Sigma$. If an edge's label is $(x, y)$, one calls $x$ the input and $y$ the output, and denotes the transition by $p \stackrel{x \mid y}{\longrightarrow} q$. Such a Mealy automaton $\mathcal{M}$ is assumed to be complete and deterministic in its inputs: for every state $p$ and letter $x$, there exists exactly one transition from $p$ with input letter $x$. We denote by $x^{p}$ its corresponding output letter and by $p @ x$ its target state, so we have

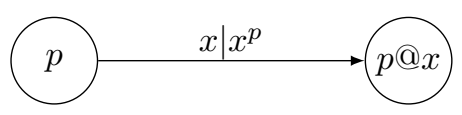

In this way, states act on letters and letters on states. Such actions can be composed in the following way: let $\mathbf{q} \in Q^{*}, p \in Q, \mathbf{u} \in \Sigma^{*}$, and $x \in \Sigma$, we have

$$
x^{\mathbf{q} p}=\left(x^{\mathbf{q}}\right)^{p} \quad \text { and } \quad p @(\mathbf{u} x)=(p @ \mathbf{u}) @ x .
$$

We extend recursively the actions of states on letters and of letters on states (see below left). Compositions can be more easily understood via an alternative representation by a cross-diagram [1] (below right).

For all $x \in \Sigma, \mathbf{u} \in \Sigma^{*}, p \in Q, \mathbf{q} \in Q^{*}$, we have: 


$$
\begin{aligned}
& (\mathbf{u} x)^{\mathbf{q}}=\mathbf{u}^{\mathbf{q}} x^{\mathbf{q} @ \mathbf{u}} \\
& \text { and }
\end{aligned}
$$

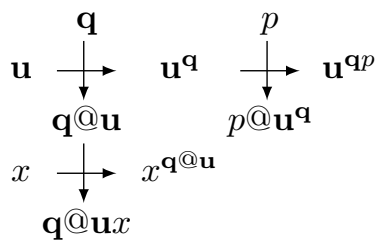

The mappings defined above are length-preserving and prefix-preserving. Note that in particular the image of the empty word is itself.

From an algebraic point of view, the composition gives a semigroup structure to the set of transformations $\mathbf{u} \mapsto \mathbf{u}^{\mathbf{q}}$ for $\mathbf{q} \in Q^{*}$. This semigroup is called the semigroup generated by $\mathcal{M}$ and denoted by $\langle\mathcal{M}\rangle_{+}$. An automaton semigroup is a semigroup which can be generated by a Mealy automaton. Any element of such an automaton semigroup induces a so-called finite-state transformation.

Conversely, for any transformation $t$ of $\Sigma^{*}$ and any word $\mathbf{u} \in \Sigma^{*}$, we denote by $\mathbf{u}^{t}$ the image of $\mathbf{u}$ by $t$, and by $t @ \mathbf{u}$ the unique transformation $s$ of $\Sigma^{*}$ satisfying $(\mathbf{u v})^{t}=\mathbf{u}^{t} \mathbf{v}^{s}$ for any $\mathbf{v} \in \Sigma^{*}$. Whenever $Q(t)=\left\{t @ \mathbf{u}: \mathbf{u} \in \Sigma^{*}\right\}$ is finite, $t$ is said to be finite-state and admits a unique (minimal) associated Mealy automaton $\mathcal{M}_{t}$ with stateset $Q(t)$.

We also use the following convenient notation to define a finite-state transformation $t$ : for each $u \in Q(t)$, we write an equation (traditionally called wreath recursion in the algebraic theory of automata) of the following form

$$
u=\left(u @ x_{1}, \ldots, u @ x_{|\Sigma|}\right) \sigma_{u},
$$

where $\sigma_{u}=\left[x_{1}^{u}, \ldots, x_{|\Sigma|}{ }^{u}\right]$ denotes the transformation on $\Sigma$ induced by $u$.

We consider the semigroup $\operatorname{FEnd}\left(\Sigma^{*}\right)$ of those finite-state transformations of $\Sigma^{*}$.

Example 2.2. The transformation $t_{0}=\left(\mathbb{1}, t_{0}\right)[2,2]$ belongs to $\operatorname{FEnd}\left(\{1,2\}^{*}\right)$ with $Q\left(t_{0}\right)=\left\{\mathbb{1}, t_{0}\right\}$. See Examples 4.4 and 5.3 for further details about $t_{0}$.

Example 2.3. The transformation $p=(q, r)$ with $q=(r, \mathbb{1})$ and $r=(r, r)[2,2]$ also belongs to $\operatorname{FEnd}\left(\{1,2\}^{*}\right)$ with $Q(p)=\{\mathbb{1}, p, q, r\}$. See Figure 1a for $\mathcal{M}_{p}$.

\section{An activity-based hierarchy for $\operatorname{FEnd}\left(\Sigma^{*}\right)$}

In this section we define a suitable notion of activity for finite-state transformations, together with two norms, from which we build a new hierarchy. We will prove its strictness and its computability in Section 4.

For any element $t \in \operatorname{FEnd}\left(\Sigma^{*}\right)$, we define its activity (see Figure 1) as

$$
\alpha_{t}: n \longmapsto \#\left\{\mathbf{v} \in \Sigma^{n}: \exists \mathbf{u} \in \Sigma^{n}, t @ \mathbf{u} \neq \mathbb{1} \text { and } \mathbf{u}^{t}=\mathbf{v}\right\} .
$$

We next define two norms on $\operatorname{FEnd}\left(\Sigma^{*}\right)$. When $\alpha_{t}$ has polynomial growth, namely when the set $D=\left\{d: \lim _{n \rightarrow \infty} \frac{\alpha_{t}(n)}{n^{d}}=0\right\}$ is nonempty, then we define $\|t\|_{\mathrm{p}}=\min D-1$. Otherwise, the value of $\lim _{n \rightarrow \infty} \frac{\log \alpha_{t}(n)}{n}$ is denoted by $\|t\|_{\mathrm{e}}$. 


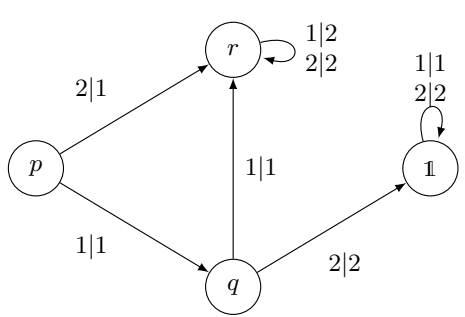

(a)

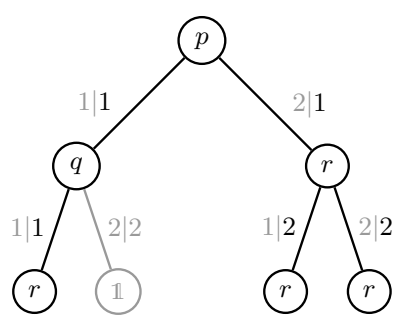

(b)

Fig. 1: (a) An example of a transformation $p$ with $\alpha_{p}(1)=1$ and $\alpha_{p}(2)=2$. (b) The transformation $p$ induces 3 nontrivial transformations on level 2: the leftmost one is associated with the output 11, the middle right one with 12 and the rightmost one with 12 , hence nontrivial transformations can be reached by runs with only two different output words.

We then define the following classes of finite-state transformations:

$$
\begin{aligned}
\operatorname{SPol}(d) & =\left\{t \in \operatorname{FEnd}\left(\Sigma^{*}\right):\|t\|_{\mathrm{p}} \leq d\right\} \\
\text { and } \operatorname{SExp}(\lambda) & =\left\{t \in \operatorname{FEnd}\left(\Sigma^{*}\right):\|t\|_{\mathrm{e}} \leq \lambda\right\} .
\end{aligned}
$$

We shall see in Theorem 3.3 that these yield a strict and computable hierarchy for $\operatorname{FEnd}\left(\Sigma^{*}\right)$. The following basic lemma is crucial:

Lemma 3.1. For each $n \geq 0$, the map $t \mapsto \alpha_{t}(n)$ is subadditive.

Proof. Assume $s, t \in \operatorname{FEnd}\left(\Sigma^{*}\right)$.

For any $\mathbf{u} \in \Sigma^{n}$ with $(s t) @ \mathbf{u} \neq \mathbb{1}$, we have either $s @ \mathbf{u} \neq \mathbb{1}$ or $t @ \mathbf{u}^{s} \neq \mathbb{1}$. We deduce $\alpha_{s t}(n) \leq \alpha_{s}(n)+\alpha_{t}(n)$ for each $n \geq 0$.

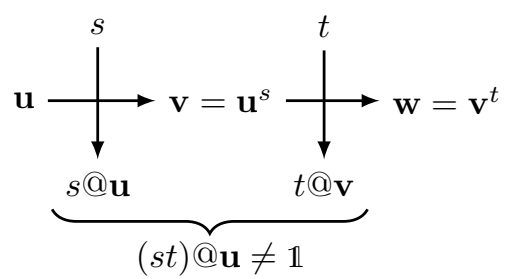

We deduce that $\|\cdot\|_{\mathrm{p}}$ and $\|\cdot\|_{\mathrm{e}}$ are respectively +- and max-subadditive.

Proposition 3.2. Let $\Sigma$ be an alphabet. For every integer $d \geq-1, \operatorname{SPol}(d)$ is a subsemigroup of $\operatorname{FEnd}\left(\Sigma^{*}\right)$. So is $\operatorname{SExp}(\lambda)$ for every $0 \leq \lambda \leq \# \Sigma$.

As an easy corollary of Proposition 3.2, the subadditivity property allows us to compute the hierarchy class of any given Mealy automaton by considering only its generators.

Theorem 3.3. Let $\Sigma$ be an alphabet. The elements of the semigroup $\operatorname{FEnd}\left(\Sigma^{*}\right)$ can be graded according to the following strict hierarchy: for any $d_{1}, d_{2} \in \mathbb{Z}$ with $-1<d_{1}<d_{2}$ and any $\lambda_{1}, \lambda_{2} \in \mathbb{R}$ with $0<\lambda_{1}<\lambda_{2}<\# \Sigma$, we have:

$$
\begin{gathered}
\operatorname{SPol}(-1) \subsetneq \cdots \subsetneq \operatorname{SPol}\left(d_{1}\right) \subsetneq \cdots \subsetneq \operatorname{SPol}\left(d_{2}\right) \subsetneq \cdots \subsetneq \operatorname{SExp}(0) \\
\subsetneq \operatorname{SExp}\left(\lambda_{1}\right) \subsetneq \cdots \subsetneq \operatorname{SExp}\left(\lambda_{2}\right) \subsetneq \cdots \subsetneq \operatorname{SExp}(\# \Sigma) .
\end{gathered}
$$


The proof of the previous result is postponed to the end of Section 4 on page 8 .

Sidki defined in [19] the activity of a finite-state automorphism $t \in \operatorname{FAut}\left(\Sigma^{*}\right)$ as

$$
\theta_{t}: n \longmapsto \#\left\{\mathbf{u} \in \Sigma^{n}: t @ \mathbf{u} \neq \mathbb{1}\right\}
$$

and the corresponding classes $\operatorname{Pol}(d)$. Using this notion of activity $\theta$ for transformations leads inevitably to an impasse: the associated classes with fixed degree polynomial $\theta$-activity would be not closed under composition. However it is straightforward that our new notion of activity $\alpha$ coincides with Sidki's activity $\theta$ in the case of automorphisms.

The class $\operatorname{SExp}(0)$ coincides with the infinite union $\bigcup_{d \geq-1} \operatorname{SPol}(d)$, whose corresponding automorphisms class is denoted by $\operatorname{Pol}(\infty)$ in [19].

\section{Structural characterization of the activity norm}

From [6], we know that the finite-state automorphisms which have polynomial activity are exactly those whose underlying automaton does not contain entangled cycles (except on the trivial state). Moreover, the degree of the polynomial is given by the longest chain of cycles in the automaton. The first claim remains true for transformations, but things are a bit more involved for the second one (see Example 4.4).

To any minimal Mealy automaton $\mathcal{M}$ with stateset $Q$ and alphabet $\Sigma$, we associate its pruned output automaton $\mathcal{M}^{\text {out }}$ defined as the NFA with stateset $Q \backslash\{\mathbb{1}\}$ (all states being final) and alphabet $\Sigma$, and whose transitions are given, for $p, q \in Q \backslash\{\mathbb{1}\}$, by

$$
p \stackrel{y}{\longrightarrow} q \in \mathcal{M}^{\text {out }} \Longleftrightarrow p \stackrel{x \mid y}{\longrightarrow} q \in \mathcal{M} \text {. }
$$

According to context, we shall identify a transformation $t \in \operatorname{FEnd}\left(\Sigma^{*}\right)$, the state of $\mathcal{M}_{t}$, and the corresponding state of $\mathcal{M}_{t}^{\text {out }}$.

Lemma 4.1. The activity of a transformation $t \in \operatorname{FEnd}\left(\Sigma^{*}\right)$ is the number of paths starting from $t$ in the (non-complete) deterministic automaton $\operatorname{det}\left(\mathcal{M}_{t}^{\text {out }}\right)$ constructed via the Rabin-Scott construction.

Proof. Let $t \in \operatorname{FEnd}\left(\Sigma^{*}\right)$ with $\mathcal{M}_{t}$ its associated automaton. Let us count the words $\mathbf{v} \in \Sigma^{n}$ for which there is a word $\mathbf{u} \in \Sigma^{n}$ with $t @ \mathbf{u} \neq \mathbb{1}$ and $\mathbf{u}^{t}=\mathbf{v}$. For $n=1, \alpha_{t}(1)$ is exactly the number of different outputs from the state $t$ that do not lead to a trivial state of $\mathcal{M}_{t}$. Now for $\mathbf{v} \in \Sigma^{n}$, if $\mathcal{E}$ denotes the set of those states accessible from $t$ by reading $\mathbf{v}$ (this corresponds to the Rabin-Scott powerset construction) in $\mathcal{M}_{t}^{\text {out }}$, the number of ways to extend $\mathbf{v}$ without getting into a trivial state in $\mathcal{M}_{t}$ corresponds to the number of outputs of the state $\mathcal{E}$ in $\operatorname{det}\left(\mathcal{M}_{t}^{\text {out }}\right)$, whence the result. 
Whether the activity of a given $t \in \operatorname{FEnd}\left(\Sigma^{*}\right)$ is polynomial or exponential can be decided by only looking at the cycle structure of $\mathcal{M}_{t}^{\text {out }}$. Any cycle considered throughout this paper is simple: no repetitions of vertices or edges are allowed. Two cycles are coreachable if there exists a path from any of them to the other one. A chain of cycles is a sequence of cycles such that each cycle is reachable from its predecessor.

Proposition 4.2. A transformation $t \in \mathrm{FEnd}\left(\Sigma^{*}\right)$ has exponential activity if and only if it can reach two coreachable cycles with distinct labels in $\mathcal{M}_{t}^{\text {out }}$.

Proof. $(\Leftarrow)$ Assume that $t$ can reach a state $s \in \mathcal{M}_{t}^{\text {out }}$ that lies on two cycles with distinct labels. There exist a word $\mathbf{u} \in \Sigma^{*}$ satisfying $t @ \mathbf{u}=s$ and two words $\mathbf{v}, \mathbf{w} \in \Sigma^{*}$ satisfying $s @ \mathbf{v}=s=s @ \mathbf{w}$ and $\mathbf{v}^{s} \neq \mathbf{w}^{s}$. We obtain $\alpha_{t}(\ell) \geq$ $\#\left\{\mathbf{x}^{t} \mid \mathbf{x} \in \mathbf{u}(\mathbf{v}+\mathbf{w})^{*} \cap \Sigma^{\ell}\right\}$ for $\ell \geq 0$. Therefore $\alpha_{t}$ grows exponentially.

$(\Rightarrow)$ Assume that $t$ has exponential activity and cannot reach two coreachable cycles in $\mathcal{M}_{t}^{\text {out }}$. By Lemma 4.1, there exist a subset $\mathcal{E} \subset Q$ and three words $\mathbf{u}, \mathbf{v}, \mathbf{w} \in \Sigma^{*}$ such that $\mathcal{E}$ is the set of nontrivial states accessible from $t$ reading $\mathbf{u}$ and the paths labeled by $\mathbf{v}$ and $\mathbf{w}$ are cycles starting from $\mathcal{E}$ in $\mathcal{M}_{t}^{\text {out }}$. It means that $\mathbf{v}$ and $\mathbf{w}$ are also cycles starting from $t$ in $\mathcal{M}_{t}$, contradiction.

Using the subadditivity of the activity (see Lemma 3.1), we get for the polynomial activities:

Corollary 4.3. Let $\mathcal{M}$ be a Mealy automaton. The transformations of $\langle\mathcal{M}\rangle_{+}$ are all of polynomial activity if and only if there are no coreachable cycles in the automaton $\operatorname{det}\left(\mathcal{M}^{\text {out }}\right)$. Moreover the degree of the (polynomial) activity corresponds to the longest chain of cycles in $\operatorname{det}\left(\mathcal{M}^{\text {out }}\right)$ minus 1.

Example 4.4. Consider the transformation $t_{0}=\left(\mathbb{1}, t_{0}\right)[2,2]$ from Example 2.2, its square $t_{0}^{2}$, and the associated automata $\mathcal{M}_{t_{0}^{2}}$ and $\operatorname{det}\left(\mathcal{M}_{t_{0}^{2}}^{\text {out }}\right)$ :
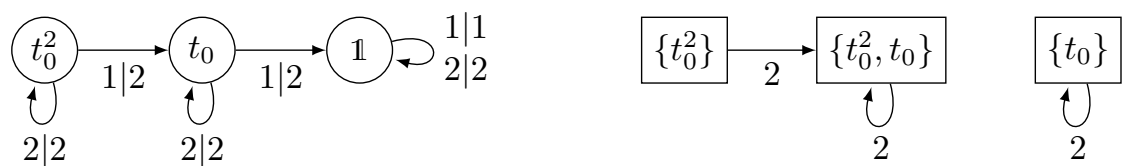

Note that, before determinization, two disjoint cycles are accessible from the state $t_{0}^{2}$. In the determinized version, $\left\{t_{0}\right\}$ and $\left\{t_{0}^{2}\right\}$ both access to only one cycle, and we conclude $\left\{t_{0}, t_{0}^{2}\right\} \subset \operatorname{SPol}(0)$. By Proposition 3.2, we actually knew the full inclusion $\left\langle t_{0}\right\rangle_{+} \subset \operatorname{SPol}(0)$.

Defining further $t_{k}=\left(t_{k-1}, t_{k}\right)[2-(k \bmod 2), 2-(k \bmod 2)] \in \operatorname{FEnd}\left(\{1,2\}^{*}\right)$, we obtain the family with $t_{k} \in \operatorname{SPol}(k) \backslash \operatorname{SPol}(k-1)$ for $k>0$, that witnesses the strictness of the polynomial part of the hierarchy from Theorem 3.3.

Using Proposition 2.1, we obtain an explicit formula for the norm $\|\cdot\|_{\mathrm{e}}$ : 
Proposition 4.5. Let $t$ be a finite-state transformation with associated Mealy automaton $\mathcal{M}_{t}$. The norm $\|t\|_{\mathrm{e}}$ is the logarithm of the Perron eigenvalue of the transition matrix of $\operatorname{det}\left(\mathcal{M}_{t}^{\text {out }}\right)$ :

$$
\|t\|_{\mathrm{e}}=\log \lambda\left(\operatorname{det}\left(\mathcal{M}_{t}^{\text {out }}\right)\right) .
$$

Proof. By Lemma 4.1, the activity of $t$ counts the number of paths in $\operatorname{det}\left(\mathcal{M}_{t}^{\text {out }}\right)$. Since all its states are final, this automaton is coaccessible and the cardinality of the language accepted when putting $t$ as the initial state is exactly the activity of $t$. Therefore by Proposition 2.1, we have

$$
\|t\|_{\mathrm{e}}=\lim _{\ell \rightarrow \infty} \frac{\log \alpha_{t}(\ell)}{\ell}=\lim _{\ell \rightarrow \infty} \frac{1}{\ell} \log \sum_{t^{\prime}=1}^{n}\left(A^{\ell}\right)_{t, t^{\prime}}=h(L)=\log \lambda\left(\operatorname{det}\left(\mathcal{M}_{t}^{\text {out }}\right)\right),
$$

with $A=\left(A_{i, j}\right)_{i, j}$ the adjacency matrix of $\operatorname{det}\left(\mathcal{M}_{t}^{\text {out }}\right)$.

Proof of Theorem 3.3. The strictness for the polynomial part is obtained from Example 4.4. Now, as the norm $\|\cdot\|_{\mathrm{e}}$ is the logarithm of the maximal eigenvalue of a matrix with integer coefficients, the classes $\operatorname{SExp}(\lambda)$ increase only when $e^{\lambda}$ is an algebraic integer that is the largest zero of its minimal polynomial, i.e., a root of a Perron number. Furthermore, each of these numbers is the norm of some finite-state transformation, see [12, Theorem 3] for a proof. It is also known that Perron numbers are dense in $[1, \infty)$, which gives us the strictness for the exponential part: $\lambda_{1}<\lambda_{2}$ implies $\operatorname{SExp}\left(\lambda_{1}\right) \subsetneq \operatorname{SExp}\left(\lambda_{2}\right)$.

Finally, the growth rate can be computed with any precision $0<\delta<1$ in time $\Theta(-\log (\delta n))$, where $n$ is the number of states of the automaton [17].

Example 4.6. Consider the transformations $r=(s, r)[1,1]$ and $s=(\mathbb{1}, r)$ with common associated automata $\mathcal{M}$ (on the left) and $\operatorname{det}\left(\mathcal{M}^{\text {out }}\right)$ (on the right):

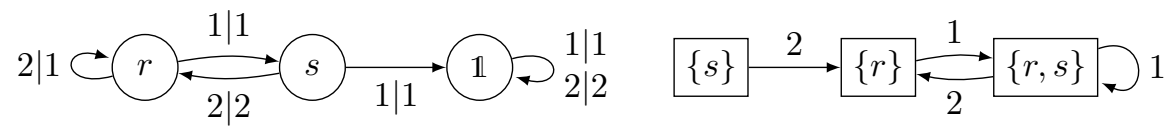

We find that $\alpha_{r}(n)$ and $\alpha_{s}(n+1)$ correspond to the $n$-th Fibonacci number. We deduce $\|r\|_{\mathrm{e}}=\|s\|_{\mathrm{e}}=\log \varphi$ where $\varphi$ is the golden ratio, hence $r, s \in \operatorname{SExp}(\log \varphi)$.

\section{The orbit signalizer graph and the order problem}

This section is devoted to the ORDER Problem: can one decide whether a given element generates a finite semigroup? The latter is known to be undecidable for general automaton semigroups [8] and decidable for $\operatorname{Pol}(0)[6]$. We give a general construction that associates a graph to a transformation of $\Sigma^{*}$, and show that, if finite, this graph lets us compute the index and period of the transformation. We show that this graph is finite for elements from $\mathrm{SPol}(0)$, and solve the ORDER Problem in this manner. 
Let $\Sigma$ be an alphabet. We define the orbit signalizer graph $\Phi$ for $\operatorname{FEnd}\left(\Sigma^{*}\right)$ as the following (infinite) graph. The vertices are the pairs of elements in $\operatorname{FEnd}\left(\Sigma^{*}\right)$. For each letter $x \in \Sigma$, there is an arrow from the source $(s, t)$ with label $(x: m, \ell)$ where $m$ and $\ell$ are the minimal integers (with $\ell>0$ ) satisfying

$$
x^{s t^{m+\ell}}=x^{s t^{m}}
$$

and with target $\left(r @ x, t^{\ell} @ x^{r}\right)$ for $r=s t^{m}$. The parameters $m$ and $\ell$ correspond respectively to the index and to the period of the orbit of $x$ under the action of $s t^{\omega}$, see Figure 2 .

In what follows, the intuition is roughly to generalize Figure 2, by considering a path $\pi$ instead of the letter $x$ : such a construction leads also to a pan graph, whose handle has length between $i_{t}^{-}$and $i_{t}^{+}$, and whose cycle has length $p_{t}$. The main challenge here is to be able to keep the construction finite, when possible.

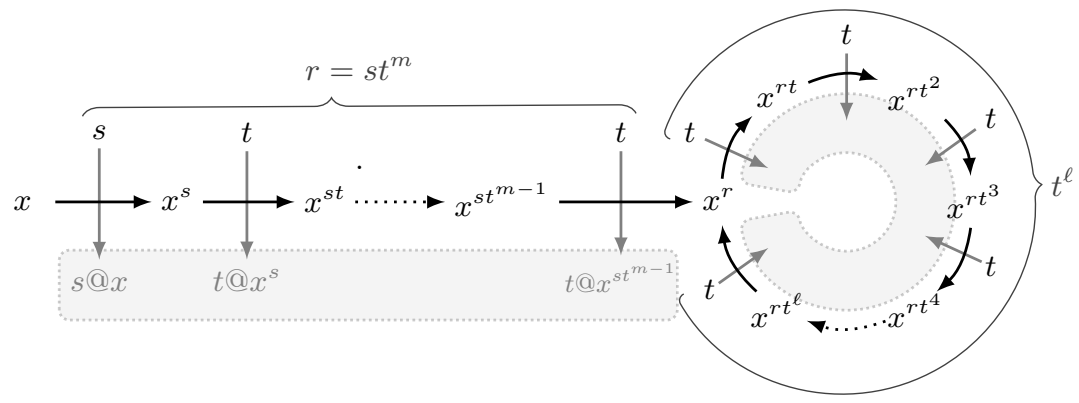

Fig. 2: The cross-diagram associated with the orbit of some letter $x \in \Sigma$ under the action of $s^{\omega}$. The index $m$ and period $\ell$ will complete the label of the $x$-arrow away from the vertex $(s, t)$ in the graph $\Phi$. Each of the two gray zones indicates an entry of the corresponding target vertex $\left(r @ x, t^{\ell} @ x^{r}\right)$ with $r=s t^{m}$.

The inf-index-cost, sup-index-cost, and the period-cost of a given walk $\pi$ on $\Phi$

$$
\pi:(s, t) \stackrel{x_{1}: m_{1}, \ell_{1}}{\longrightarrow} \cdots \stackrel{x_{|\pi|}: m_{|\pi|}, \ell_{|\pi|}}{\longrightarrow}\left(s^{\prime}, t^{\prime}\right)
$$

are respectively defined by

$$
\begin{aligned}
& i^{-}(\pi)=\sum_{1 \leq k \leq|\pi|}\left(\left(1-\delta_{m_{k}, 0}\right)\left(\left(m_{k}-1\right)\left(\prod_{1 \leq j<k} \ell_{j}\right)+1\right)\right), \\
& i^{+}(\pi)=\sum_{1 \leq k \leq|\pi|}\left(\prod_{1 \leq j<k} \ell_{j}\right) m_{k}, \quad \text { and } \quad p(\pi)=\prod_{1 \leq i \leq|\pi|} \ell_{i} .
\end{aligned}
$$

For any $t \in \operatorname{FEnd}\left(\Sigma^{*}\right)$, we define the orbit signalizer graph $\Phi(t)$ as the subgraph of $\Phi$ accessible from the source vertex $(\mathbb{1}, t)$. The inf-index-cost, sup-index-cost, 
and the period-cost of $t \in \operatorname{FEnd}\left(\Sigma^{*}\right)$ are then respectively defined by

$$
i_{t}^{-}=\max _{\pi \text { on } \Phi(t)} i^{-}(\pi), \quad i_{t}^{+}=\max _{\pi \text { on } \Phi(t)} i^{+}(\pi), \quad \text { and } \quad p_{t}=\operatorname{lcm}_{\pi \text { on } \Phi(t)} p(\pi) .
$$

Proposition 5.1. The semigroup generated by an element $t \in \operatorname{FEnd}\left(\Sigma^{*}\right)$ is finite if and only if its index-costs $i_{t}^{ \pm}$and its period-cost $p_{t}$ are finite. In that case, we have $\langle t\rangle_{+}=\left\langle t: t^{i_{t}}=t^{i_{t}+p_{t}}\right\rangle_{+}$for some index $i_{t}$ with $i_{t}^{-} \leq i_{t} \leq i_{t}^{+}$.

Proof. Let $\Sigma=\left\{x_{1}, \ldots, x_{|\Sigma|}\right\}$. Let $\left(s_{0}, t_{0}\right)$ be a vertex in $\Phi$ and $\left(s_{k}, t_{k}\right)$ its successor vertex with arrow $x_{k}: m_{k}, \ell_{k}$ for $1 \leq k \leq|\Sigma|$.

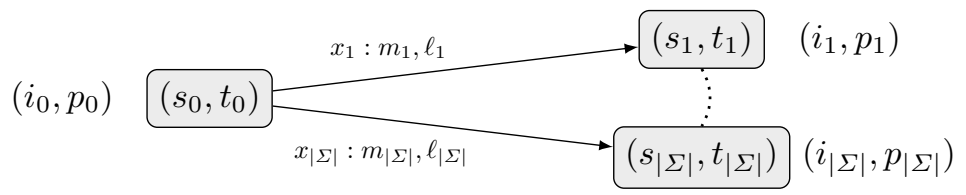

For $0 \leq k \leq|\Sigma|$, let $\left(i_{k}, p_{k}\right) \in\{\omega, 0,1,2, \ldots\} \times\{\omega, 1,2,3 \ldots\}$ denote the possible minimal pair of ordinals (with $p_{k}>0$ ) satisfying

$$
s_{k} t_{k}^{i_{k}}=s_{k} t_{k}^{i_{k}+p_{k}} .
$$

Whenever there is at least one successor with $\left(i_{k}, p_{k}\right)=(\omega, \omega),\left(s_{0}, t_{0}\right)$ satisfies also $\left(i_{0}, p_{0}\right)=(\omega, \omega)$, and so does any of its predecessors. Otherwise, we claim

$$
\max _{1 \leq k \leq|\Sigma|}\left(m_{k}+\max \left(0, \ell_{k}\left(i_{k}-1\right)+1\right)\right) \leq i_{0} \leq \max _{1 \leq k \leq|\Sigma|}\left(m_{k}+\ell_{k} i_{k}\right)
$$

and

$$
p_{0}=\operatorname{lcm}_{1 \leq k \leq|\Sigma|} \ell_{k} p_{k}
$$

Indeed, for $1 \leq k \leq|\Sigma|$ and for any $u \in \Sigma^{*}$, we have

$$
y_{k} v=\left(x_{k} u\right)^{s_{0} t_{0}^{m_{k}+\ell_{k} i_{k}}}=\left(y_{k} v\right)^{t_{0}^{\ell_{k} p_{k}}}
$$

with $y_{k}=x_{k}{ }^{s_{0} t_{0}^{m_{k}}}$ and $v=u^{s_{k} t_{k}^{i_{k}+p_{k}}}$, as illustrated by the cross-diagram:

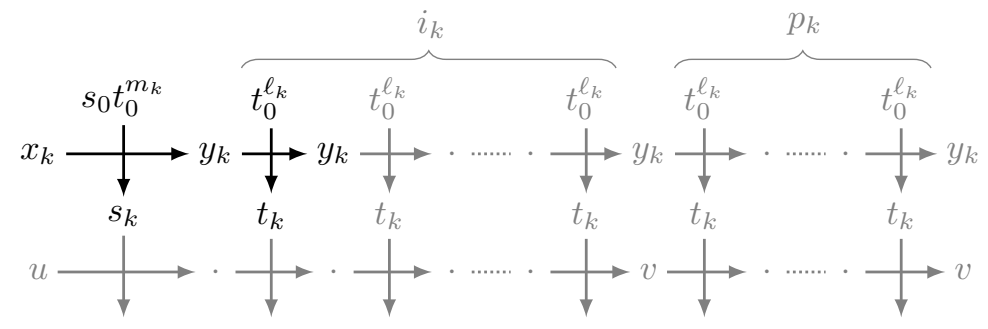

We conclude using an induction on the length of the paths.

Theorem 5.2. The ORder Problem is decidable for any $t \in \operatorname{FEnd}\left(\Sigma^{*}\right)$ with a finite orbit signalizer graph $\Phi(t)$. 
Proof. Since $\Phi(t)$ is a graph with outdegree $\|\Sigma\|>0$ by construction, its finiteness implies the existence of cycles. Consider the simple cycles (there is only a finite number of these). One can compute the index-costs $i^{-}(\kappa)$ and $i^{+}(\kappa)$ and the period-cost $p(\kappa)$ of each such cycle $\kappa$. Whenever $i^{-}(\kappa)>0$ or $p(\kappa)>1$ for some cycle $\kappa$, then $t$ has infinite order, and finite order otherwise.

Example 5.3. The transformations $s=(s, \mathbb{1})[2,2]$ and $t_{0}=\left(\mathbb{1}, t_{0}\right)[2,2]$ (on the left) admit respective graphs $\Phi(s)$ and $\Phi\left(t_{0}\right)$ (on the right):
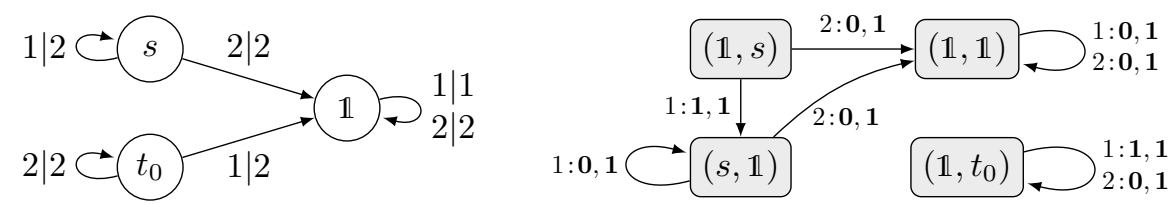

According to Proposition 5.1, they generate the finite monoid $\left\langle s: s^{2}=s\right\rangle_{+}$ and the free monoid $\left\langle t_{0}:\right\rangle_{+}$.

Example 5.4. The transformation $b=(a, 1, b)[2,3,1]$ from $\operatorname{SPol}(1) \backslash \operatorname{SPol}(0)$ with $a=(\mathbb{1}, \mathbb{1}, a)[1,1,2]$ admits the finite graph $\Phi(b)$ displayed on Figure 3, in which we can read that both $a b$ and $b a$ have period 1, and that $b$ has thus period $p_{b}=3$. According to Proposition 5.1 again, the index of $b$ satisfies $4 \leq$ $i_{b} \leq 9$, and can be explicitly computed as $i_{b}=8$.

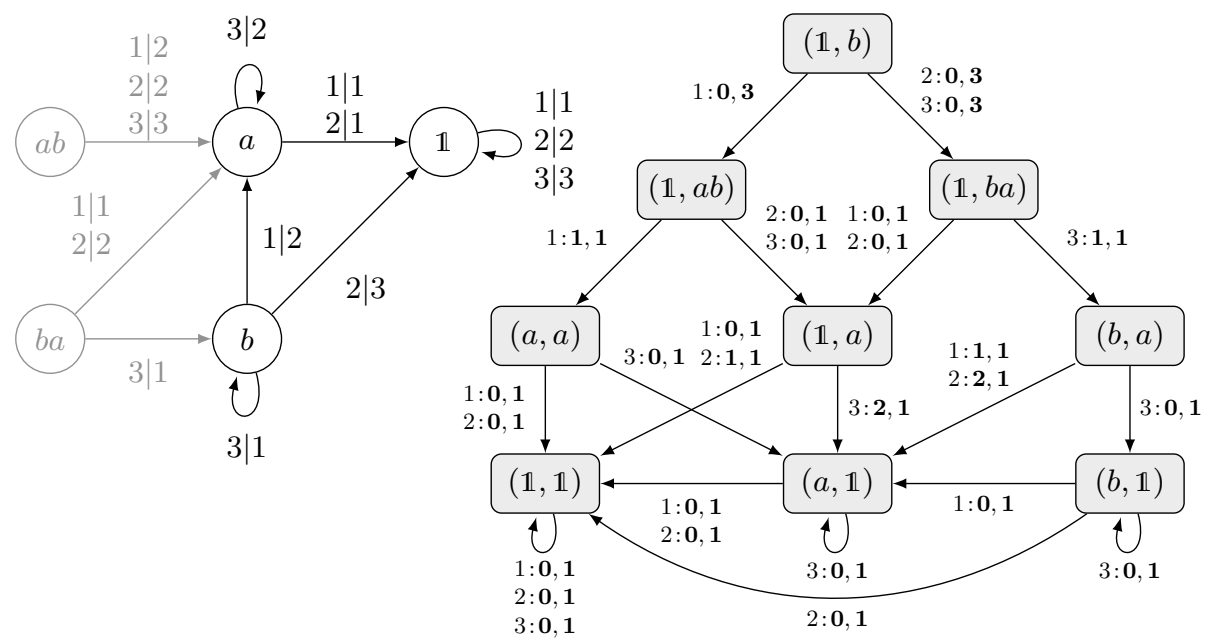

Fig. 3: The Mealy automaton $\mathcal{M}_{b}$ and the graph $\Phi(b)$ from Example 5.4.

Proposition 5.5. Every bounded finite-state transformation $t \in \mathrm{SPol}(0)$ admits a finite orbit signalizer graph $\Phi(t)$. 
Proof. The activity $\alpha_{t}$ of $t \in \operatorname{SPol}(0)$ is uniformly bounded by some constant $C$ :

$$
\#\left\{\mathbf{v} \in \Sigma^{n}: \exists \mathbf{u} \in \Sigma^{n}, t @ \mathbf{u} \neq \mathbb{1} \text { and } \mathbf{u}^{t}=\mathbf{v}\right\} \leq C \text { for } n \geq 0 .
$$

Now the vertices of the graph $\Phi(t)$ are those pairs $(r, s)$ with $r s=t^{K} @ \mathbf{u}$, where $K$ is the greatest integer such that the images $\mathbf{u}^{t^{i}}$ for $i<K$ are pairwise different. Hence $r s$ is the product of at most $C$ nontrivial elements. Moreover these elements $t^{i} @ \mathbf{u}$ for $i<K$ lie in a finite set, as $t$ is a finite-state transformation, hence the vertices belong to a finite set. We conclude that the graph $\Phi(t)$ is finite.

Corollary 5.6. The Order Problem is decidable for SPol(0).

\section{References}

1. A. Akhavi, I. Klimann, S. Lombardy, J. Mairesse, and M. Picantin, The finiteness problem for automaton semigroups, Internat. J. Alg. Comput. 22(6) (2012), 1-26.

2. L. Bartholdi, Algorithmic decidability of Engel's property for automaton groups, CSR 2016, LNCS, vol. 9691, pp. 29-40.

3. _ FR - GAP package "Computations with functionally recursive groups", Version 2.4.3, 2017, http://www.gap-system.org/Packages/fr.html.

4. L. Bartholdi and I. Mitrofanov, The word and order problems for self-similar and automata groups, 2017, arXiv:1710.10109.

5. L. Bartholdi and P. V. Silva, Groups defined by automata, AutoMathA Handbook (J.-É. Pin, ed.), Europ. Math. Soc., 2018, arXiv:1012.1531.

6. I. Bondarenko, N. Bondarenko, S. Sidki, and F. Zapata, On the conjugacy problem for finite-state automorphisms, Groups Geom. Dyn. 7(2) (2013), 323-355.

7. N. Chomsky and G. A. Miller, Finite state languages, Information and Control 1(2) (1958), 91-112.

8. P. Gillibert, The finiteness problem for automaton semigroups is undecidable, Internat. J. Alg. Comput. 24(1) (2014), 1-9.

9. __ An automaton group with undecidable order and Engel problems, J. Algebra 497 (2018), 363-392.

10. Th. Godin and I. Klimann, On bireversible Mealy automata and the Burnside problem, Theor. Comput. Sci. 707 (2018), 24-35.

11. I. Klimann, J. Mairesse, and M. Picantin, Implementing computations in automaton (semi)groups, CIAA 2012, LNCS, vol. 7381, pp. 240-252.

12. D. A. Lind, The entropies of topological markov shifts and a related class of algebraic integers, Ergodic Theory and Dynamical Systems 4(2) (1984), 283-300.

13. D. A. Lind and B. Marcus, An introduction to symbolic dynamics and coding, Cambridge University Press, 1995.

14. M. O. Rabin and D. Scott, Finite automata and their decision problems, IBM Journal of Research and Development 3(2) (1959), 114-125.

15. M. Rigo, Advanced graph theory and combinatorics, Wiley, 2016.

16. J. Sakarovitch, Elements of automata theory, Cambridge University Press, 2009.

17. A. M. Shur, Combinatorial complexity of regular languages, CSR'08, LNCS, vol. 5010, 2008, pp. 289-301.

18. - Comparing complexity functions of a language and its extendable part, RAIRO-Theoretical Informatics and Applications 42(3) (2008), 647-655.

19. S. Sidki, Automorphisms of one-rooted trees: growth, circuit structure, and acyclicity, Journal of Mathematical Sciences 100(1) (2000), 1925-1943. 